\title{
Rapid computation of far-field statistics for random obstacle scattering
}

\author{
Helmut Harbrecht \\ Helmut Harbrecht, Departement Mathematik und Informatik, Universität Basel, \\ Spiegelgasse 1, 4051 Basel, Schweiz. \\ Nikola Ilić \\ Nikola Ilić, Departement Mathematik und Informatik, Universität Basel, Spiegelgasse 1, \\ 4051 Basel, Schweiz. \\ Michael D. Multerer \\ Michael D. Multerer, Institute of Computational Science, USI Lugano, Via Giuseppe \\ Buffi 13, 6900 Lugano, Schweiz
}

\begin{abstract}
In this article, we consider the numerical approximation of far-field statistics for acoustic scattering problems in the case of random obstacles. In particular, we consider the computation of the expected far-field pattern and the expected scattered wave away from the scatterer as well as the computation of the corresponding variances. To that end, we introduce an artificial interface, which almost surely contains all realizations of the random scatterer. At this interface, we directly approximate the second order statistics, i.e., the expectation and the variance, of the Cauchy data by means of boundary integral equations. From these quantities, we are able to rapidly evaluate statistics of the scattered wave everywhere in the exterior domain, including the expectation and the variance of the far-field. By employing a low-rank approximation of the Cauchy data's two-point correlation function, we drastically reduce the cost of the computation of the scattered wave's variance. Numerical results are provided in order to demonstrate the feasibility of the
\end{abstract}

Email addresses: helmut.harbrecht@unibas.ch (Helmut Harbrecht), n.ilic@unibas.ch (Nikola Ilić), michael.multerer@usi.ch (Michael D. Multerer) 
proposed approach.

\section{Introduction}

The propagation of an acoustic wave in a homogeneous, isotropic, and inviscid fluid is approximately described by a velocity potential $U(\mathbf{x}, t)$ satisfying the wave equation

$$
U_{t t}=c^{2} \Delta U
$$

Here, $c$ denotes the speed of sound, $\mathbf{v}=\nabla U$ is the velocity field, and $p=-U_{t}$ is the pressure, see [5, Chapter 3] for instance. If $U$ is time harmonic, that is

$$
U(\mathbf{x}, t)=\operatorname{Re}\left(u(\mathbf{x}) e^{-i \omega t}\right), \quad \omega>0,
$$

in complex notation, then the complex-valued space-dependent function $u$ satisfies the Helmholtz equation

$$
\Delta u+\kappa^{2} u=0 \quad \text { in } \mathbb{R}^{d} \backslash \bar{D},
$$

where $D \subset \mathbb{R}^{d}, d=2,3$, corresponds to an obstacle and $\kappa=\omega / c$ is the wavenumber. We assume that $D$ is a bounded and simply connected domain, having a smooth boundary $\Gamma=\partial D$. For sound-soft obstacles the pressure $p$ vanishes on $\Gamma$, which leads to the Dirichlet boundary condition

$$
u=0 \quad \text { on } \Gamma \text {. }
$$

We shall consider the situation that the total wave

$$
u=u_{\text {inc }}+u_{\mathrm{s}}
$$

is comprised of a known incident plane wave $u_{\text {inc }}(\mathbf{x})=e^{i \kappa\langle\mathbf{d}, \mathbf{x}\rangle}$ with direction $\mathbf{d} \in \mathbb{R}^{d}$, where $\|\mathbf{d}\|_{2}=1$, and the scattered wave $u_{\mathrm{s}}$. Then, if we impose the Sommerfeld radiation condition

$$
\sqrt{r}\left(\frac{\partial u_{\mathrm{s}}}{\partial r}-i \kappa u_{\mathrm{s}}\right) \rightarrow 0 \text { as } r:=\|\mathbf{x}\|_{2} \rightarrow \infty,
$$

for the scattered wave, we obtain a unique solution to the acoustic scattering problem

$$
\begin{aligned}
\Delta u+\kappa u & =0 & & \text { in } \mathbb{R}^{d} \backslash \bar{D} \\
u & =0 & & \text { on } \Gamma \\
\sqrt{r}\left(\frac{\partial u_{\mathrm{s}}}{\partial r}-i \kappa u_{\mathrm{s}}\right) & \rightarrow 0 & & \text { as } r=\|\mathbf{x}\|_{2} \rightarrow \infty
\end{aligned}
$$


see [5, Chapter 3]. In particular, the Sommerfeld radiation condition implies the asymptotic behavior

$$
u_{\mathrm{s}}(\mathbf{x})=\frac{e^{i \kappa r}}{r}\left\{u_{\infty}\left(\frac{\mathbf{x}}{r}\right)+\mathcal{O}\left(\frac{1}{r}\right)\right\}, \quad r \rightarrow \infty .
$$

Herein, the function

$$
u_{\infty}: \mathbb{S}^{1}:=\left\{\hat{\mathbf{x}} \in \mathbb{R}^{d}:\|\hat{\mathbf{x}}\|_{2}=1\right\} \rightarrow \mathbb{C}
$$

is called the far-field pattern, which is always analytic in accordance with [5, Chapter 6].

In this article, we consider the situation that the scatterer $D$ is randomly shaped, i.e., $D=D(\mathbf{y})$ for a random parameter $\mathbf{y} \in \square:=[-1,1]^{\mathbb{N}}$. Hence, the scattered wave itself becomes a random field $u_{\mathrm{s}}(\mathbf{y})$. We will model a class of random domains and compute the associated expected scattered wave $\mathbb{E}\left[u_{\mathrm{s}}\right]$ and also the expected far-field $\mathbb{E}\left[u_{\infty}\right]$. Instead of employing the domain mapping method, which maps the deformed scatterer onto a fixed reference domain, as in e.g. [4, 7, 12, 15, 16, 23], or a fictitious domain approach as in [3], we will compute all samples for the deformed scatterer by means of the boundary element method. This approach is much cheaper since we do not require a very fine triangulation for $D$ in order to ensure that the domain deformation field is properly resolved. Consequently, we are also able to deal with large variations without the need of a very fine discretization.

Furthermore, we derive a means to compute the scattered wave's second order statistics in a deterministic fashion from its Cauchy data's second order statistics on an artificial, deterministic interface $\Sigma$, which almost surely contains the domain $D(\mathbf{y})$. By the application of a low-rank approximation for the correlation function, we are able to considerably decrease the cost for the computation of the expected scattered field and its variance. The advantages of the proposed approach are thus as follows:

(i) The use of boundary integral equations facilitates a straightforward treatment of the unbounded exterior domain. Especially, it avoids expensive mesh generation procedures in case of strongly varying scatterers.

(ii) Since the artificial interface is bounded and has one dimension less than to the exterior domain, the impact of the high dimensionality of the random scattering problem is drastically reduced. 
We like to emphasize that the present approach is also suitable to treat sound-hard scatterers, where the Dirichlet boundary condition in (1.2) becomes a Neumann condition. In addition, scatterers with a different diffractive index $\kappa$ can be considered. The latter leads to a transmission condition at the scatterer's surface instead of a boundary condition. The presented ideas remain valid in this situation except for modifying the boundary integral equations accordingly. Moreover, although we focus on $d=2$ in the numerical examples, all concepts can be transferred to $d=3$ in a straightforward manner. However, technicalities will increase.

The rest of the article is organized as follows. In Section 2, we introduce the formulation of the scattering problem under consideration in the case of a deterministic scatterer by means of boundary integral equations. In particular, we provide a representation of the total wave and the far-field pattern. Then, in Section 3, we consider a representation of random scatterers in terms of random vector fields. Moreover, we provide an explicit description for the two dimensional situation, which is used later on in the numerical examples. Section 4 deals with the random scattering problem. Here, we derive expressions for the scattered wave's expectation and variance, including the far-field pattern. Section 5 is dedicated to numerical results which quantify and qualify our approach. The boundary integral equations are discretized by the Nyström method which converges exponentially in case of analytic boundaries. Especially, we discuss the efficient computation of the scattered wave's variance by using a low-rank approximation. Finally, in Section 6, we state concluding remarks.

\section{Boundary integral representation of the scattering problem}

\subsection{Computing the scattered wave}

We shall recall the solution of the boundary value problem (1.2) by means of boundary integral equations. For the sake of simplicity in representation, we assume here that the domain $D$ is fixed with a smooth boundary $\Gamma=\partial D$.

We introduce the acoustic single layer operator

$$
\mathcal{V}: H^{-1 / 2}(\Gamma) \rightarrow H^{1 / 2}(\Gamma), \quad \mathcal{V} \rho:=\int_{\Gamma} \Phi(\cdot, \mathbf{z}) \rho(\mathbf{z}) \mathrm{d} \sigma_{\mathbf{z}}
$$

and the acoustic double layer operator

$$
\mathcal{K}: L^{2}(\Gamma) \rightarrow L^{2}(\Gamma), \quad \mathcal{K} \rho:=\int_{\Gamma} \frac{\partial \Phi(\cdot, \mathbf{z})}{\partial \mathbf{n}_{\mathbf{z}}} \rho(\mathbf{z}) \mathrm{d} \sigma_{\mathbf{z}}
$$


Herein, $\Phi(\cdot, \cdot)$ denotes the fundamental solution of the Helmholtz equation. It is given by

$$
\Phi\left(\mathbf{x}, \mathbf{x}^{\prime}\right)= \begin{cases}\frac{i}{4} H_{0}^{(1)}\left(\kappa\left\|_{\mathbf{x}}-\mathbf{x}^{\prime}\right\|_{2}\right), & d=2 \\ \frac{e^{i \kappa\left\|\mathbf{x}-\mathbf{x}^{\prime}\right\|_{2}}}{4 \pi\left\|\mathbf{x}-\mathbf{x}^{\prime}\right\|_{2}}, & d=3\end{cases}
$$

where $H_{0}^{(1)}$ denotes the zeroth order Hankel function of the first kind.

Then, if the incident wave is given by

$$
u_{\text {inc }}(\mathbf{x})=e^{i \kappa\langle\mathbf{d}, \mathbf{x}\rangle}
$$

for some direction $\mathbf{d} \in \mathbb{R}^{d}$, the Neumann data of the total wave $u=u_{\text {inc }}+u_{\mathrm{s}}$ at the boundary $\Gamma$ can be determined by the boundary integral equation

$$
\left(\frac{1}{2}+\mathcal{K}^{\star}-i \eta \mathcal{V}\right) \frac{\partial u}{\partial \mathbf{n}}=\frac{\partial u_{\text {inc }}}{\partial \mathbf{n}}-i \eta u_{\text {inc }} \quad \text { on } \Gamma
$$

where $\mathcal{K}^{\star}$ denotes the adjoint double-layer operator, $\mathbf{n}$ the outward pointing normal vector and $\eta \in \mathbb{R} \backslash\{0\}$ is chosen such that $\eta \operatorname{Re}(\kappa)>0$, see $[1,5]$.

From the Cauchy data of $u$ at $\Gamma$, we can determine the scattered wave $u_{\mathrm{s}}$ in any point in the exterior of the scatterer by applying the potential evaluation

$$
u_{\mathrm{s}}(\mathbf{x})=\int_{\Gamma} \Phi(\mathbf{x}, \mathbf{z}) \frac{\partial u}{\partial \mathbf{n}}(\mathbf{z}) \mathrm{d} \sigma_{\mathbf{z}}, \quad \mathbf{x} \in \mathbb{R}^{d} \backslash \bar{D} .
$$

By letting $\|\mathbf{x}\|_{2}$ tend to infinity in (2.2), we derive a closed expression for the far-field of the total wave $u$. Namely, the far-field at a point $\widehat{\mathbf{x}} \in \mathbb{S}^{1}$ is given in accordance with

$$
u_{\infty}(\hat{\mathbf{x}})=\int_{\Gamma} \Phi_{\infty}(\hat{\mathbf{x}}, \mathbf{z}) \frac{\partial u}{\partial \mathbf{n}}(\mathbf{z}) \mathrm{d} \sigma_{\mathbf{z}}
$$

Herein, the far-field kernel $\Phi_{\infty}(\cdot, \cdot)$ is given according to

$$
\Phi_{\infty}(\hat{\mathbf{x}}, \mathbf{z})= \begin{cases}\frac{e^{i \pi / 4}}{\sqrt{8 \kappa \pi}} e^{-i \kappa\langle\hat{\mathbf{x}}, \mathbf{z}\rangle}, & d=2, \\ \frac{1}{4 \pi} e^{-i \kappa\langle\hat{\mathbf{x}}, \mathbf{z}\rangle}, & d=3 .\end{cases}
$$




\subsection{Alternative representation of the scattered wave}

We shall introduce the sphere

$$
\Sigma:=\left\{\mathbf{x} \in \mathbb{R}^{d}:\|\mathbf{x}\|_{2}=R\right\}
$$

of radius $R>0$, being sufficiently large to guarantee that $\Sigma$ encloses the domain $D$. By differentiating (2.2), it is seen that the gradient of the scattered wave can simply be computed by

$$
\nabla u_{\mathrm{s}}(\mathbf{x})=\int_{\Gamma} \nabla_{\mathbf{x}} \Phi(\mathbf{x}, \mathbf{z}) \frac{\partial u}{\partial \mathbf{n}}(\mathbf{z}) \mathrm{d} \sigma_{\mathbf{z}}, \quad \mathbf{x} \in \Sigma .
$$

Thus, we can compute the Cauchy data of the scattered wave at the artificial interface $\Sigma$. Especially, it holds

$$
\frac{\partial u_{\mathrm{s}}}{\partial \mathbf{n}}(\mathbf{x})=\int_{\Gamma} \frac{\partial \Phi(\mathbf{x}, \mathbf{z})}{\partial \mathbf{n}_{\mathbf{x}}} \frac{\partial u}{\partial \mathbf{n}}(\mathbf{z}) \mathrm{d} \sigma_{\mathbf{z}}, \quad \mathbf{x} \in \Sigma,
$$

where $\mathbf{n}_{\mathbf{x}}=\mathbf{x} /\|\mathbf{x}\|_{2}$ is the outward pointing normal at $\mathbf{x} \in \Sigma$.

For any $\mathbf{x} \in \mathbb{R}^{d}$ with $\|\mathbf{x}\|_{2}>R$, we can now either use the representation formula (2.2) or the representation formula

$$
u_{\mathrm{s}}(\mathbf{x})=\int_{\Sigma}\left\{\Phi(\mathbf{x}, \mathbf{z}) \frac{\partial u_{\mathrm{s}}}{\partial \mathbf{n}}(\mathbf{z})+\frac{\partial \Phi(\mathbf{x}, \mathbf{z})}{\partial \mathbf{n}_{\mathbf{z}}} u_{\mathrm{s}}(\mathbf{z})\right\} \mathrm{d} \sigma_{\mathbf{z}}
$$

to evaluate the scattered wave $u_{\mathrm{s}}$ at any point $\mathbf{x} \in \mathbb{R}^{d}$ with $\|\mathbf{x}\|_{2}>R$. In particular, letting $R \rightarrow \infty$, we obtain for the far-field the formula

$$
u_{\infty}(\hat{\mathbf{x}})=\int_{\Sigma}\left\{\Phi_{\infty}(\hat{\mathbf{x}}, \mathbf{z}) \frac{\partial u}{\partial \mathbf{n}}(\mathbf{z})+\frac{\partial \Phi_{\infty}(\mathbf{x}, \mathbf{z})}{\partial \mathbf{n}_{\mathbf{z}}} u(\mathbf{z})\right\} \mathrm{d} \sigma_{\mathbf{z}}, \quad \hat{\mathbf{x}} \in \mathbb{S}^{1}
$$

As we will see, the major advantage of (2.4) and (2.5) over (2.2) and (2.3) in case of a random scatterer is that the sphere $\Sigma$ has a fixed shape.

We remark that an artificial interface being different from a circle can of course be chosen as well.

\section{Random obstacles}

\subsection{Representation of random domains}

In this section, we introduce a description of random obstacles by means of random vector fields, as they have originally been considered in [12] in the 
context of the domain mapping method. To that end, let $(\Omega, \mathcal{A}, \mathbb{P})$ denote a complete and separable probability space with $\sigma$-algebra $\mathcal{A}$ and probability measure $\mathbb{P}$. Here, complete means that $\mathcal{A}$ contains all $\mathbb{P}$-null sets. For a given real or complex Banach space $\mathcal{X}$, we introduce the Lebesgue-Bochner space $L_{\mathbb{P}}^{p}(\Omega ; \mathcal{X}), 1 \leq p \leq \infty$, which consists of all equivalence classes of strongly measurable functions $v: \Omega \rightarrow \mathcal{X}$ with bounded norm

$$
\|v\|_{L_{\mathbb{P}}^{p}(\Omega ; \mathcal{X})}:= \begin{cases}\left(\int_{\Omega}\|v(\cdot, \omega)\|_{\mathcal{X}}^{p} \mathrm{~d} \mathbb{P}(\omega)\right)^{1 / p}, & p<\infty, \\ \operatorname{ess~sup}_{\omega \in \Omega}\|v(\cdot, \omega)\|_{\mathcal{X}}, & p=\infty .\end{cases}
$$

If $p=2$ and $\mathcal{X}$ is a separable Hilbert space, then the Lebesgue-Bochner space $L_{\mathbb{P}}^{p}(\Omega ; \mathcal{X})$ is isomorphic to the tensor product space $L_{\mathbb{P}}^{2}(\Omega) \otimes \mathcal{X}$. For more details on Lebesgue-Bochner spaces, we refer the reader to [14].

For $p \geq 2$ and a given random field $v \in L^{p}(\Omega ; \mathcal{X})$, we can introduce the expectation

$$
\mathbb{E}[v](\mathbf{x}):=\int_{\Omega} v(\mathbf{x}, \omega) \mathrm{d} \mathbb{P}(\omega)
$$

and the variance

$$
\mathbb{V}[v](\mathbf{x}):=\int_{\Omega} v(\mathbf{x}, \omega) \overline{v(\mathbf{x}, \omega)} \mathrm{d} \mathbb{P}(\omega)-\mathbb{E}[v](\mathbf{x}) \overline{\mathbb{E}[v](\mathbf{x})} .
$$

With straighforward modifications, these definitions remain valid for real valued random fields.

Now, to define the random obstacle $D(\omega) \subset \mathbb{R}^{d}$, we assume the existence of a nominal obstacle $D_{0} \subset \mathbb{R}^{d}$, with boundary $\Gamma_{0}:=\partial D_{0}$, and of a uniform $C^{1}$-diffeomorphism

$$
\mathbf{V}: \overline{D_{0}} \times \Omega \rightarrow \mathbb{R}^{d}
$$

i.e. there holds

$$
\|\mathbf{V}(\omega)\|_{C^{1}\left(\overline{D_{0}} ; \mathbb{R}^{d}\right)},\left\|\mathbf{V}^{-1}(\omega)\right\|_{C^{1}\left(\overline{D_{0}} ; \mathbb{R}^{d}\right)} \leq C_{\text {uni }} \quad \text { for } \mathbb{P} \text {-a.e. } \omega \in \Omega,
$$

such that $D(\omega)$ is implicitly given by the relation

$$
D(\omega)=\mathbf{V}\left(D_{0}, \omega\right)
$$

Consequently, we obtain

$$
\Gamma(\omega):=\partial D(\omega)=\mathbf{V}\left(\Gamma_{0}, \omega\right)
$$


Moreover, we define the hold-all domain $\mathcal{D}$ according to

$$
\mathcal{D}:=\bigcup_{\omega \in \Omega} D(\omega) .
$$

Due to (3.1), it holds $\mathbf{V} \in L^{\infty}\left(\Omega ; C^{1}\left(\overline{D_{0}}\right)\right) \subset L^{2}\left(\Omega ; C^{1}\left(\overline{D_{0}}\right)\right)$. Hence, the vector field $\mathbf{V}$ can be represented by a Karhunen-Loève expansion, cf. [18], of the form

$$
\mathbf{V}(\mathbf{x}, \omega)=\mathbb{E}[\mathbf{V}](\mathbf{x})+\sum_{k=1}^{\infty} \mathbf{V}_{k}(\mathbf{x}) Y_{k}(\omega) .
$$

The Karhunen-Loève expansion separates spatial and random contributions of the random vector field by means of the spatial vector fields $\left\{\mathbf{V}_{k}\right\}_{k}$ and the uncorrelated random variables $\left\{Y_{k}\right\}_{k}$.

An efficient way to compute the Karhunen-Loève expansion if the mean and the covariance function of the random (vector) field under consideration are known is given by the pivoted Cholesky decomposition. This accounts particularly for random vector fields, see [10, 11, 19].

The anisotropy which is induced by the spatial parts $\left\{\mathbf{V}_{k}\right\}_{k}$, describing the fluctuations around the nominal value $\mathbb{E}[\mathbf{V}](\mathbf{x})$ in (3.3), is encoded by the quantities

$$
\lambda_{k}:=\left\|\mathbf{V}_{k}\right\|_{W^{1, \infty}\left(D_{0} ; \mathbb{R}^{d}\right)} .
$$

For our modeling, we shall also make the following common assumptions.

\section{Assumption 1.}

(i) The random variables $\left\{Y_{k}\right\}_{k}$ take values in $[-1,1]$.

(ii) The random variables $\left\{Y_{k}\right\}_{k}$ are independent and uniformly distributed, i.e. $Y_{k} \sim \mathcal{U}(-1,1)$.

(iii) The sequence $\left\{\lambda_{k}\right\}_{k}$ is at least in $\ell^{1}(\mathbb{N})$.

We remark that it holds without loss of generality $\mathbb{E}[\mathbf{V}](\mathbf{x})=\mathbf{x}$, otherwise we have to choose an appropriate reparametrization. Moreover, identifying the random variables by their image $\mathbf{y} \in \square:=[-1,1]^{\mathbb{N}}$, we end up with the representation

$$
\mathbf{V}(\mathbf{x}, \mathbf{y})=\mathbf{x}+\sum_{k=1}^{\infty} \mathbf{V}_{k}(\mathbf{x}) y_{k} .
$$


The corresponding image measure $\mu$ is given by the product of the push forward measure $\nu=\mathrm{d} y / 2$ according to $\mu:=\otimes_{k=1}^{\infty} \nu$. The Jacobian of $\mathbf{V}$ with respect to the spatial variable $\mathbf{x}$ is thus given by

$$
\mathbf{J}(\mathbf{x}, \mathbf{y})=\mathbf{I}+\sum_{k=1}^{\infty} \mathbf{V}_{k}^{\prime}(\mathbf{x}) y_{k}
$$

Herein, $\mathbf{V}_{k}^{\prime}$ denotes the Jacobian of $\mathbf{V}_{k}$ for $k=1,2, \ldots$

The uniformity condition implies that the functional determinant $\operatorname{det} \mathbf{J}(\mathbf{x}, \mathbf{y})$ is either uniformly positive or negative, see [12] for more details. Hence, we may assume without loss of generality

$$
0<c \leq \operatorname{det} \mathbf{J}(\mathbf{x}, \mathbf{y}) \leq C<\infty
$$

for every $\mathbf{x} \in D_{0}$ and almost every $\mathbf{y} \in \square$, where $c, C>0$ are some constants. For a sufficiently fine discretization of the domain $D_{0}$, this property carries over to the finite element approximation of $\mathbf{V}$ and $\mathbf{J}$, respectively. Again, we refer to [12] for the details. Hence, for a sufficiently fine mesh, i.e. the resolution is such that the uniformity condition (3.1) carries over to the discretized random vector field, a quasi-uniform mesh will be mapped to a quasi-uniform mesh for almost every $\mathbf{y} \in \square$. Consequently, no re-meshing of $\Gamma(\mathbf{y})$ is necessary. However, in view of the Nyström method we shall employ later on, this implies that we have to use a sufficient number of quadrature points at the boundary.

\subsection{Star-like obstacles in two spatial dimensions}

In the numerical examples, we will restrict ourselves to star-like scatterers in two spatial dimensions. Especially, since we consider a boundary integral approach to solve the Helmholtz equation, we shall only define here the random vector field only for the boundary $\Gamma_{0}$.

Without loss of generality, we assume that the scatterer $D(\omega)$ is star-like with respect to the origin $\mathbf{0} \in \mathbb{R}^{2}$. Then, we can represent its boundary $\Gamma(\omega)$ by a parametrization of the form

$$
\gamma:[0,2 \pi] \times \Omega \rightarrow \mathbb{R}^{2}, \quad \gamma(\phi)=r(\phi, \omega) \mathbf{e}_{r}(\phi)
$$

where

$$
\mathbf{e}_{r}(\phi)=\left[\begin{array}{l}
\cos (\phi) \\
\sin (\phi)
\end{array}\right]
$$


denotes the radial direction and radius function $r(\phi, \omega)$ is a real valued random field $r \in L^{2}\left(\Omega ; C_{\text {per }}^{n}([0,2 \pi])\right)$. As before, the Karhunen-Loève expansion of $r$ can be computed if $\mathbb{E}[r](\phi)$ and

$$
\operatorname{Cov}[r]\left(\phi, \phi^{\prime}\right):=\int_{\Omega}(r(\phi, \omega)-\mathbb{E}[r](\phi))\left(r\left(\phi^{\prime}, \omega\right)-\mathbb{E}[r]\left(\phi^{\prime}\right)\right) \mathrm{d} \mathbb{P}(\omega) .
$$

are known. However, we will assume here that $r$ is of the particular form

$$
r(\phi, \mathbf{y})=r_{0}(\phi)+\sum_{k=1}^{\infty}\left\{a_{2 k-1} y_{2 k-1} \sin (k \phi)+a_{2 k} y_{2 k} \cos (k \phi)\right\}, \quad \mathbf{y} \in \square .
$$

In this case, the spatial regularity is entirely encoded in the coefficients $a_{k}$, $k \geq 0$, see [15]. By construction, the random fluctuations of the radius (3.7) are centered, i.e., their mean vanishes, and we conclude

$$
\mathbb{E}[r](\phi)=\int_{\square} r(\phi, \mathbf{y}) \mathrm{d} \mu=r_{0}(\phi)
$$

In order to guarantee that each realization $\mathbf{y} \in[-1,1]^{\mathbb{N}}$ results in a boundary of a valid domain $D(\mathbf{y})$, we shall further assume that

$$
0<\underline{r} \leq r(\phi, \mathbf{y}) \leq \bar{r}<\infty \quad \text { for all } \phi \in[0,2 \pi] \text { and } \mathbf{y} \in \square \text {. }
$$

Moreover, it is assumed that $r_{0} \in C_{\mathrm{per}}^{2}([0,2 \pi])$ as well as that the sequence $\left(a_{k}\right)_{k}$ decays sufficiently fast to ensure $r(\cdot, \mathbf{y}) \in C_{\text {per }}^{2}([0,2 \pi])$ for all $\mathbf{y} \in \square$. This for example is guaranteed if $\left|a_{k}\right| \leq c k^{-(3+\varepsilon)}$ for any $\varepsilon>0$ and some constant $c>0$.

The random boundary $\Gamma(\mathbf{y})$ is hence given by

$$
\Gamma(\mathbf{y})=\left\{\gamma(\phi, \mathbf{y})=r(\phi, \mathbf{y}) \mathbf{e}_{r}(\phi) \in \mathbb{R}^{2}: \phi \in[0,2 \pi]\right\}
$$

Consequently, there holds $\left.\mathbf{V}\right|_{\Gamma_{0}}=\boldsymbol{\gamma}$, where $\Gamma_{0}=r_{0} \mathbf{e}_{r}$. In order to provide a description of the random vector field for the interior of the scatterer, which is for example needed in a domain mapping approach, a suitable extension of $\mathbf{V}$ to $D_{0}$ has to be defined as in e.g. $[12,15]$. However, we remark that in the approach presented here, no knowledge of $\mathbf{V}$ inside the scatterer is required. 


\section{The random scattering problem}

\subsection{Problem formulation}

Now, having a suitable description of the random scatterer $D(\mathbf{y})$ at our disposal, we can define the random scattering problem under consideration. Let $u_{\text {inc }}$ denote the incident wave. Then, the boundary value problem for the total field $u(\mathbf{y})=u_{\mathrm{s}}(\mathbf{y})+u_{\text {inc }}$ for a given $\mathbf{y} \in \square$ reads

$$
\begin{array}{rlrl}
\Delta u(\mathbf{y})+\kappa u(\mathbf{y})=0 & & \text { in } \mathbb{R}^{d} \backslash \overline{D(\mathbf{y})} \\
u(\mathbf{y})=0 & & \text { on } \Gamma(\mathbf{y}) \\
\sqrt{r}\left(\frac{\partial u_{\mathrm{s}}}{\partial r}-i \kappa u_{\mathrm{s}}\right) & \rightarrow 0 & & \text { as } r=\|\mathbf{x}\|_{2} \rightarrow \infty .
\end{array}
$$

By the construction of $\Gamma(\mathbf{y})$, the random scattering problem (4.1) exhibits a unique solution for each realization $\mathbf{y} \in \square$ of the random parameter. Moreover, it has been shown in [15] for the case of the Helmholtz transmission problem in two spatial dimensions that the total wave $u(\mathbf{y})$ in the large wavelength regime exhibits an analytic extension with respect to the parameter $\mathbf{y} \in \square$ into a certain region of the complex plane. Hence, given that $\kappa$ is sufficiently small, we may employ higher order quadrature methods, like the quasi-Monte Carlo methods, see e.g. [2], or sparse quadrature methods, see e.g. $[9,15]$, for the computation of quantities of interest.

\subsection{Expected scattered wave}

We can compute the scattered wave's expectation for a given point $\mathbf{x} \in \mathbb{R}^{d}$ via the potential evaluation (2.2), which leads to

$$
\mathbb{E}\left[u_{\mathrm{s}}\right](\mathbf{x})=\mathbb{E}\left[\int_{\Gamma(\mathbf{y})} \Phi(\mathbf{x}, \mathbf{z}) \frac{\partial u_{\mathrm{s}}}{\partial \mathbf{n}}(\mathbf{z}, \cdot) \mathrm{d} \sigma_{\mathbf{z}}\right] .
$$

Of course, (4.2) makes only sense if $\mathbf{x} \notin \mathcal{D}$ since otherwise there might be instances $\mathbf{y} \in \square$ such that $\mathbf{x} \in D(\mathbf{y})$, i.e., the point $\mathbf{x}$ does not lie outside the scatterer almost surely, compare (3.2). In what follows, we assume that $R>0$ is chosen such that

$$
\mathcal{D} \subset B_{R}(\mathbf{0}):=\left\{\mathbf{x} \in \mathbb{R}^{d}:\|x\|_{2}<R\right\} \quad \text { and set } \quad \Sigma=\partial B_{R}(\mathbf{0}) .
$$

Then, if we want to compute the expectation in many points, it is much more efficient to exploit the artificial but fixed boundary $\Sigma$ and to consider 
an expression similar to (2.4). However, we remark that an application of the mapping approach as in [15] is better suited if statistics of the scattered wave in the vicinity of the scatterer are of interest.

For any $\mathbf{x} \in \mathbb{R}^{d}$ with $\|\mathbf{x}\|_{2}>R$, it holds

$$
\mathbb{E}\left[u_{\mathrm{s}}\right](\mathbf{x})=\int_{\Sigma}\left\{\Phi(\mathbf{x}, \mathbf{z}) \mathbb{E}\left[\frac{\partial u_{\mathrm{s}}}{\partial \mathbf{n}}\right](\mathbf{z})+\frac{\partial \Phi(\mathbf{x}, \mathbf{z})}{\partial \mathbf{n}_{\mathbf{z}}} \mathbb{E}\left[u_{\mathrm{s}}\right](\mathbf{z})\right\} \mathrm{d} \sigma_{\mathbf{z}}
$$

Therefore, the scattered wave's expectation is completely encoded in the Cauchy data at the artificial boundary $\Sigma$. This means that we only need to compute the expected Cauchy data

$$
\mathbb{E}\left[u_{\mathrm{s}}\right]=\int_{\square}\left\{\int_{\Gamma(\mathbf{y})} \Phi(\mathbf{x}, \mathbf{z}) \frac{\partial u}{\partial \mathbf{n}}(\mathbf{z}, \mathbf{y}) \mathrm{d} \sigma_{\mathbf{z}}\right\} \mathrm{d} \mu
$$

and

$$
\mathbb{E}\left[\frac{\partial u_{\mathrm{s}}}{\partial \mathbf{n}}\right]=\int_{\square}\left\{\int_{\Gamma(\mathbf{y})} \frac{\partial \Phi(\mathbf{x}, \mathbf{z})}{\partial \mathbf{n}_{\mathbf{z}}} u(\mathbf{z}, \mathbf{y}) \mathrm{d} \sigma_{\mathbf{z}}\right\} \mathrm{d} \mu
$$

of the scattered wave at the artificial boundary $\Sigma$.

In complete analogy to (4.3), the expected far-field pattern is likewise computed by using (2.5):

$$
\mathbb{E}\left[u_{\infty}\right](\hat{\mathbf{x}})=\int_{\Sigma}\left\{\Phi_{\infty}(\hat{\mathbf{x}}, \mathbf{z}) \mathbb{E}\left[\frac{\partial u_{\mathrm{s}}}{\partial \mathbf{n}}(\mathbf{z})\right]+\frac{\partial \Phi_{\infty}(\mathbf{x}, \mathbf{z})}{\partial \mathbf{n}_{\mathbf{z}}} \mathbb{E}\left[u_{\mathrm{s}}\right](\mathbf{z})\right\} \mathrm{d} \sigma_{\mathbf{z}} .
$$

\subsection{Computing the solution's variance}

The variance $\mathbb{V}\left[u_{\mathrm{s}}\right]$ of the scattered wave $u_{\mathrm{s}}$ at a point $\mathbf{x} \notin B_{R}(\mathbf{0})$ depends nonlinearly on the Cauchy data of $u_{\mathrm{s}}$ at the artificial interface $\Sigma$. Nonetheless, we can employ the fact that the variance is the trace of the covariance function:

$$
\mathbb{V}\left[u_{\mathrm{s}}\right](\mathbf{x})=\left.\operatorname{Cov}\left[u_{\mathrm{s}}\right]\left(\mathbf{x}, \mathbf{x}^{\prime}\right)\right|_{\mathbf{x}=\mathbf{x}^{\prime}}=\left.\operatorname{Cor}\left[u_{\mathrm{s}}\right]\left(\mathbf{x}, \mathbf{x}^{\prime}\right)\right|_{\mathbf{x}=\mathbf{x}^{\prime}}-\left|\mathbb{E}\left[u_{\mathrm{s}}\right](\mathbf{x})\right|^{2} .
$$

The covariance function is given by

$$
\begin{aligned}
& \operatorname{Cov}\left[u_{\mathrm{s}}\right]\left(\mathbf{x}, \mathbf{x}^{\prime}\right)=\mathbb{E}\left[\left(u_{\mathrm{s}}(\mathbf{x}, \cdot)-\mathbb{E}\left[u_{\mathrm{s}}\right](\mathbf{x})\right) \overline{\left(u_{\mathrm{s}}\left(\mathbf{x}^{\prime}, \cdot\right)-\mathbb{E}\left[u_{\mathrm{s}}\right]\left(\mathbf{x}^{\prime}\right)\right)}\right] \\
& =\mathbb{E}\left[u_{\mathrm{s}}(\mathbf{x}, \cdot) \overline{u_{\mathrm{s}}\left(\mathbf{x}^{\prime}, \cdot\right)}\right]-\mathbb{E}\left[u_{\mathrm{s}}\right](\mathbf{x}) \overline{\mathbb{E}\left[u_{\mathrm{s}}\right]\left(\mathbf{x}^{\prime}\right)},
\end{aligned}
$$

and, hence,

$$
\operatorname{Cor}\left[u_{\mathrm{s}}\right]\left(\mathbf{x}, \mathbf{x}^{\prime}\right)=\mathbb{E}\left[u_{\mathrm{s}}(\mathbf{x}, \cdot) \overline{u_{\mathrm{s}}\left(\mathbf{x}^{\prime}, \cdot\right)}\right]
$$


Hence, the two-point correlation function is a higher-dimensional object. Fortunately, it depends only linearly on the second moments of the Cauchy data of the scattered wave on the artificial interface $\Sigma$, which greatly simplifies its computation. Namely, defining for $\mathbf{x}, \mathbf{x}^{\prime} \in \Sigma$ the quantities

$$
\begin{aligned}
& \operatorname{Cor}\left[u_{\mathrm{s}}\right]\left(\mathbf{x}, \mathbf{x}^{\prime}\right) \\
& =\mathbb{E}\left[\left(\int_{\Gamma(\mathbf{y})} \Phi(\mathbf{x}, \mathbf{z}) \frac{\partial u_{\mathrm{s}}}{\partial \mathbf{n}}(\mathbf{z}, \mathbf{y}) \mathrm{d} \sigma_{\mathbf{z}}\right) \overline{\left(\int_{\Gamma(\mathbf{y})} \Phi\left(\mathbf{x}^{\prime}, \mathbf{z}\right) \frac{\partial u_{\mathrm{s}}}{\partial \mathbf{n}}(\mathbf{z}, \mathbf{y}) \mathrm{d} \sigma_{\mathbf{z}}\right)}\right], \\
& \operatorname{Cor}\left[\frac{\partial u_{\mathrm{s}}}{\partial \mathbf{n}}\right]\left(\mathbf{x}, \mathbf{x}^{\prime}\right) \\
& =\mathbb{E}\left[\left(\int_{\Gamma(\mathbf{y})} \frac{\partial \Phi(\mathbf{x}, \mathbf{z})}{\partial \mathbf{n}_{\mathbf{z}}} u_{\mathrm{s}}(\mathbf{z}, \mathbf{y}) \mathrm{d} \sigma_{\mathbf{z}}\right) \overline{\left.\left(\int_{\Gamma(\mathbf{y})} \frac{\partial \Phi\left(\mathbf{x}^{\prime}, \mathbf{z}\right)}{\partial \mathbf{n}_{\mathbf{z}}} u_{\mathrm{s}}(\mathbf{z}, \mathbf{y}) \mathrm{d} \sigma_{\mathbf{z}}\right)\right]}\right.
\end{aligned}
$$

and

$$
\begin{aligned}
\mathbb{C o r} & {\left[u_{\mathrm{s}}, \frac{\partial u_{\mathrm{s}}}{\partial \mathbf{n}}\right]\left(\mathbf{x}, \mathbf{x}^{\prime}\right)=\overline{\mathbb{C o r}\left[\frac{\partial u_{\mathrm{s}}}{\partial \mathbf{n}}, u_{\mathrm{s}}\right]}\left(\mathbf{x}^{\prime}, \mathbf{x}\right) } \\
& =\mathbb{E}\left[\overline{\left(\int_{\Gamma(\mathbf{y})} \Phi(\mathbf{x}, \mathbf{z}) \frac{\partial u_{\mathrm{s}}}{\partial \mathbf{n}}(\mathbf{z}, \omega) \mathrm{d} \sigma_{\mathbf{z}}\right)}\left(\int_{\Gamma(\mathbf{y})} \frac{\partial \Phi\left(\mathbf{x}^{\prime}, \mathbf{z}\right)}{\partial \mathbf{n}_{\mathbf{z}}} u_{\mathrm{s}}(\mathbf{z}, \mathbf{y}) \mathrm{d} \sigma_{\mathbf{z}}\right)\right],
\end{aligned}
$$

we have for any $\mathbf{x}, \mathbf{x}^{\prime} \notin B_{R}(\mathbf{0})$ the deterministic expression

$$
\begin{aligned}
\operatorname{Cor}\left[u_{\mathrm{s}}\right]\left(\mathbf{x}, \mathbf{x}^{\prime}\right)=\int_{\Sigma} \int_{\Sigma}\{ & \Phi \\
& (\mathbf{x}, \mathbf{z}) \overline{\Phi\left(\mathbf{x}^{\prime}, \mathbf{z}^{\prime}\right)} \operatorname{Cor}\left[\frac{\partial u_{\mathrm{s}}}{\partial \mathbf{n}}\right]\left(\mathbf{z}, \mathbf{z}^{\prime}\right) \\
& +\Phi(\mathbf{x}, \mathbf{z}) \frac{\partial \Phi\left(\mathbf{x}^{\prime}, \mathbf{z}^{\prime}\right)}{\partial \mathbf{n}_{\mathbf{z}^{\prime}}} \operatorname{Cor}\left[\frac{\partial u_{\mathrm{s}}}{\partial \mathbf{n}}, u_{\mathrm{s}}\right]\left(\mathbf{z}, \mathbf{z}^{\prime}\right) \\
& +\frac{\partial \Phi(\mathbf{x}, \mathbf{z})}{\partial \mathbf{n}_{\mathbf{z}}} \overline{\Phi\left(\mathbf{x}^{\prime}, \mathbf{z}^{\prime}\right)} \operatorname{Cor}\left[u_{\mathrm{s}}, \frac{\partial u_{\mathrm{s}}}{\partial \mathbf{n}}\right]\left(\mathbf{z}, \mathbf{z}^{\prime}\right) \\
& \left.+\frac{\partial \Phi(\mathbf{x}, \mathbf{z})}{\partial \mathbf{n}_{\mathbf{z}}} \frac{\partial \Phi\left(\mathbf{x}^{\prime}, \mathbf{z}^{\prime}\right)}{\partial \mathbf{n}_{\mathbf{z}^{\prime}}} \operatorname{Cor}\left[u_{\mathrm{s}}\right]\left(\mathbf{z}, \mathbf{z}^{\prime}\right)\right\} \mathrm{d} \sigma_{\mathbf{z}^{\prime}} \mathrm{d} \sigma_{\mathbf{z}} .
\end{aligned}
$$

As we will see in the next section, this expression can efficiently be computed if a low-rank approximation of the Cauchy datas' correlations is available. 


\section{Numerical results}

\subsection{Random scatterer}

For our numerical experiments, we shall consider a kite-shaped scatterer as nominal obstacle, described by the parametrization

$$
\gamma:[0,2 \pi] \rightarrow \Gamma \subset \mathbb{R}^{2}, \quad \phi \mapsto \gamma(\phi):=\left[\begin{array}{c}
5 \cos (\phi)-3.25 \cos (2 \phi) \\
7.5 \sin (\phi)
\end{array}\right]
$$

The random boundary is then defined in accordance with

$$
\gamma(\phi, \mathbf{y})=\bar{\gamma}(\phi)+r(\phi, \mathbf{y})\left[\begin{array}{l}
\cos (\phi) \\
\sin (\phi)
\end{array}\right]
$$

where $\bar{\gamma}(\phi)$ denotes the kite-shaped boundary (5.1) and $r(\phi, \mathbf{y})$ is given by the Fourier series

$$
r(\phi, \mathbf{y})=\sum_{k=1}^{\infty} \frac{1}{k^{3}}\left\{\sin (k \phi) y_{2 k-1}+\cos (k \phi) y_{2 k}\right\} .
$$

For the numerical simulation, we truncate this series after 1000 terms.

Notice that the decay of the coefficients of the random fluctuations (5.3) are at the limit case. It would hold $r(\cdot, \mathbf{y}) \in C_{\mathrm{per}}^{2}([0,2 \pi])$ if the decay of the series $\left\{a_{k}\right\}_{k}$ was just a bit stronger. A visualization of 1000 samples of this boundary is found in Figure 1.

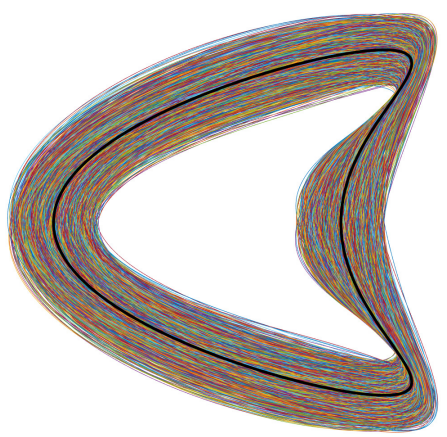

Figure 1: The kite-shaped boundary (thick black line) and 1000 random perturbations (in colour). 


\subsection{Statistics at the artificial interface}

For the numerical solution of the boundary integral equation (2.1), we apply the Nyström method to discretize the acoustic single and double layer operators. Given the parametrization (5.2) for a specific instance $\mathbf{y} \in[-1,1]^{1000}$, the method applies the trapezoidal rule in the $n=1000$ equidistantly distributed points $\varphi_{i}=2 \pi i / n, i=1, \ldots, n$, and is along the lines of $[17$, Chapter 12]. An appropriate desingularization technique based on trigonometric Lagrange polynomials is employed to deal with the singularities of the acoustic single and double layer operators. We remark that this method converges exponentially provided that the boundary under consideration is analytical. We refer the reader to [17, Chapter 12] for all the details.

We likewise subdivide the artificial interface $\Sigma=\partial B_{R}(\mathbf{0})$ by $n=1000$ equidistantly distributed points

$$
\mathbf{z}_{j}=[R \cos (2 \pi j / n), R \sin (2 \pi j / n)]^{\top}, \quad j=1, \ldots, n .
$$

In these points, we compute the expectations $\mathbb{E}\left[u_{\mathrm{s}}\right]\left(\mathbf{z}_{j}\right)$ and $\mathbb{E}\left[\partial u_{\mathrm{s}} / \partial \mathbf{n}\right]\left(\mathbf{z}_{j}\right)$ in accordance with (4.4) and (4.5), respectively. To that end, we employ the quasi-Monte Carlo method based on 10000 Halton points, cf. [20]. In [22] it is shown that this quadrature converges independent of the parameter dimension, if the derivatives with respect to the parameter $\mathbf{y} \in \square$ decay sufficiently fast. A thorough analysis of the computational work of the Nyström method in combination with the quasi-Monte Carlo quadrature can be found in [6].

In addition to the expectations of the Cauchy data, we compute the corresponding two-point correlation matrix

$$
\mathbf{C}=\left[\begin{array}{ll}
\mathbf{C}_{1,1} & \mathbf{C}_{1,2} \\
\mathbf{C}_{1,2}^{\star} & \mathbf{C}_{2,2}
\end{array}\right] \in \mathbb{C}^{2 n \times 2 n},
$$

where

$$
\mathbf{C}_{1,1}:=\left[\operatorname{Cor}\left[u_{\mathrm{s}}\right]\left(\mathbf{z}_{j}, \mathbf{z}_{j^{\prime}}\right)\right]_{j, j^{\prime}=1}^{n}, \quad \mathbf{C}_{2,2}:=\left[\operatorname{Cor}\left[\frac{\partial u_{\mathrm{s}}}{\partial \mathbf{n}}\right]\left(\mathbf{z}_{j}, \mathbf{z}_{j^{\prime}}\right)\right]_{j, j^{\prime}=1}^{n}
$$

and

$$
\mathbf{C}_{1,2}:=\left[\operatorname{Cor}\left[u_{\mathrm{s}}, \frac{\partial u_{\mathrm{s}}}{\partial \mathbf{n}}\right]\left(\mathbf{z}_{j}, \mathbf{z}_{j^{\prime}}\right)\right]_{j, j^{\prime}=1}^{n}
$$




\subsection{Low-rank approximation of the two-point correlation}

While the computation of $\mathbb{E}\left[u_{\mathrm{s}}\right](\mathbf{x})$ at a point $\mathbf{x} \notin B_{R}(\mathbf{0})$ by (4.3) is straightforward, the computation of the variance $\mathbb{V}\left[u_{\mathrm{s}}\right](\mathbf{x})$ in accordance with (4.6) amounts to the computation of $\mathbb{C o r}\left[u_{\mathrm{s}}\right](\mathbf{x}, \mathbf{x})$. This requires the approximation of the double integral over $\Sigma$. We apply again the trapezoidal rule, having thus to evaluate

$$
\begin{aligned}
\operatorname{Cor}\left[u_{\mathbf{s}}\right](\mathbf{x}, \mathbf{x}) \approx \frac{1}{(2 R \pi n)^{2}} \sum_{j, j^{\prime}=1}^{n}\{ & \Phi\left(\mathbf{x}, \mathbf{z}_{j}\right) \overline{\Phi\left(\mathbf{x}, \mathbf{z}_{j^{\prime}}\right)} \operatorname{Cor}\left[u_{\mathrm{s}}\right]\left(\mathbf{z}_{j}, \mathbf{z}_{j^{\prime}}\right) \\
& +\Phi\left(\mathbf{x}, \mathbf{z}_{j}\right) \frac{\overline{\partial \Phi\left(\mathbf{x}, \mathbf{z}_{j^{\prime}}\right)}}{\partial \mathbf{n}_{\mathbf{z}_{j^{\prime}}}} \operatorname{Cor}\left[u_{\mathrm{s}}, \frac{\partial u_{\mathrm{s}}}{\partial \mathbf{n}}\right]\left(\mathbf{z}_{j}, \mathbf{z}_{j^{\prime}}\right) \\
& +\overline{\Phi\left(\mathbf{x}, \mathbf{z}_{j}\right)} \frac{\partial \Phi\left(\mathbf{x}, \mathbf{z}_{j^{\prime}}\right)}{\partial \mathbf{n}_{\mathbf{z}_{j^{\prime}}}} \overline{\operatorname{Cor}}\left[u_{\mathrm{s}}, \frac{\partial u_{\mathrm{s}}}{\partial \mathbf{n}}\right]\left(\mathbf{z}_{j^{\prime}}, \mathbf{z}_{j}\right) \\
& \left.+\frac{\partial \Phi\left(\mathbf{x}, \mathbf{z}_{j}\right)}{\partial \mathbf{n}_{\mathbf{z}_{j}}} \frac{\overline{\partial \Phi\left(\mathbf{x}, \mathbf{z}_{j^{\prime}}\right)}}{\partial \mathbf{n}_{\mathbf{z}_{j^{\prime}}}} \operatorname{Cor}\left[\frac{\partial u_{\mathrm{s}}}{\partial \mathbf{n}}\right]\left(\mathbf{z}_{j}, \mathbf{z}_{j^{\prime}}\right)\right\} .
\end{aligned}
$$

The respective evaluations of the two-point correlation functions of the Cauchy data at $\Sigma$ are stored in the matrix $\mathbf{C}$ from (5.4). We conclude that the cost of a naïve evaluation scales quadratically in the number of degrees of freedom used at the artificial interface $\Sigma$.

In order to speed-up the computations if the variance $\mathbb{V}\left[u_{\mathrm{s}}\right](\mathbf{x})$ has to be computed in many points, we propose to compute first a low-rank approximation of the two-point correlation function of the Cauchy data at $\Sigma$. In accordance with [10], we apply the pivoted Cholesky decomposition to get a low-rank approximation

$$
\mathbf{C} \approx \mathbf{L L}^{\star}=\sum_{i=1}^{m} \boldsymbol{\ell}_{i} \ell_{i}^{\star}
$$

where $\mathbf{L}=\left[\boldsymbol{\ell}_{1}, \ldots, \boldsymbol{\ell}_{m}\right] \in \mathbb{C}^{2 n \times m}$ with $m \leq n$. Note that the truncation error can rigorously be controlled in terms of the trace. Hence, the pivoted Cholesky decomposition is truncated if

$$
\operatorname{trace}\left(\mathbf{C}-\mathbf{L L}^{\star}\right)<\varepsilon \operatorname{trace}(\mathbf{C})
$$

for some $\varepsilon>0$. For all the details, we refer to [10, 11]. We remark that we would still end up with a separable expansion if the covariance of the Cauchy data did not admit a low-rank representation. 
Having the low-rank approximation (5.5) at hand, we arrive at

$$
\mathbb{C o r}\left[u_{\mathrm{s}}\right](\mathbf{x}, \mathbf{x}) \approx \frac{1}{(2 R \pi n)^{2}} \sum_{i=1}^{m}\left|\sum_{j=1}^{n}\left[\Phi\left(\mathbf{x}, \mathbf{z}_{j}\right) \ell_{i, j}+\frac{\partial \Phi\left(\mathbf{x}, \mathbf{z}_{j}\right)}{\partial \mathbf{n}_{\mathbf{z}_{j}}} \ell_{i, n+j}\right]\right|^{2}
$$

Therefore, the evaluation of $\mathbb{C o r}\left[u_{\mathbf{s}}\right]\left(\mathbf{x}, \mathbf{x}^{\prime}\right)$ requires only $\mathcal{O}(n m)$ operations instead of $\mathcal{O}\left(n^{2}\right)$ operations. If $m \ll n$, this reduces the computational cost considerably, especially since $m$ depends only on the desired accuracy and thus only weakly on $n$.

\begin{tabular}{|c|ccccc|}
\hline \multicolumn{5}{|c|}{ rank of the low-rank approximation } \\
\hline$R$ & $\kappa=1$ & $\kappa=2$ & $\kappa=4$ & $\kappa=8$ & $\kappa=16$ \\
\hline 11 & 48 & 56 & 85 & 131 & 193 \\
12 & 39 & 51 & 83 & 131 & 194 \\
13 & 35 & 49 & 84 & 132 & 195 \\
14 & 32 & 49 & 83 & 131 & 195 \\
15 & 31 & 49 & 84 & 132 & 194 \\
\hline
\end{tabular}

Table 1: Ranks $m$ of the low-rank approximation of the two-point correlation of the Cauchy data at $\Sigma$ for varying radius $R$ and wavenumber $\kappa$.

In order to demonstrate the efficiency of the low-rank approximation, we consider again the randomly perturbed kite-shaped scatterer, given by (5.2) and (5.3). The radius of the artificial interface is varying in accordance with $R=11,12, \ldots, 15$ and the wavenumber is varying in accordance with $\kappa=1,2,4,8,16$. The number of equidistant points on $\Sigma$ is 1000 and the number of boundary elements on $\Gamma(\mathbf{y})$ is also 1000. Note that the incident wave has been chosen to come from the left, i.e., $\mathbf{d}=[1,0]^{\top}$, and the upper bound for the relative truncation error of the Cauchy data's pivoted Cholesky decomposition is $10^{-12}$, cp. (5.6). The corresponding results are found in Table 1. As can be seen, the pivoted Cholesky decomposition converges very rapidly, where the determined rank decreases for increasing $R$. In order to provide a better intuition of the Cauchy data's covariance, we have also depicted the corresponding eigenvalues for $R=11$ in Figure 2 .

\subsection{Scattered field computation}

We choose $R=11$ and compute the expectation and variance of the scattered field on the disc $\left\{\mathbf{x} \in \mathbb{R}^{2}: R \leq\|\mathbf{x}\|_{2} \leq 50\right\}$ in accordance with 

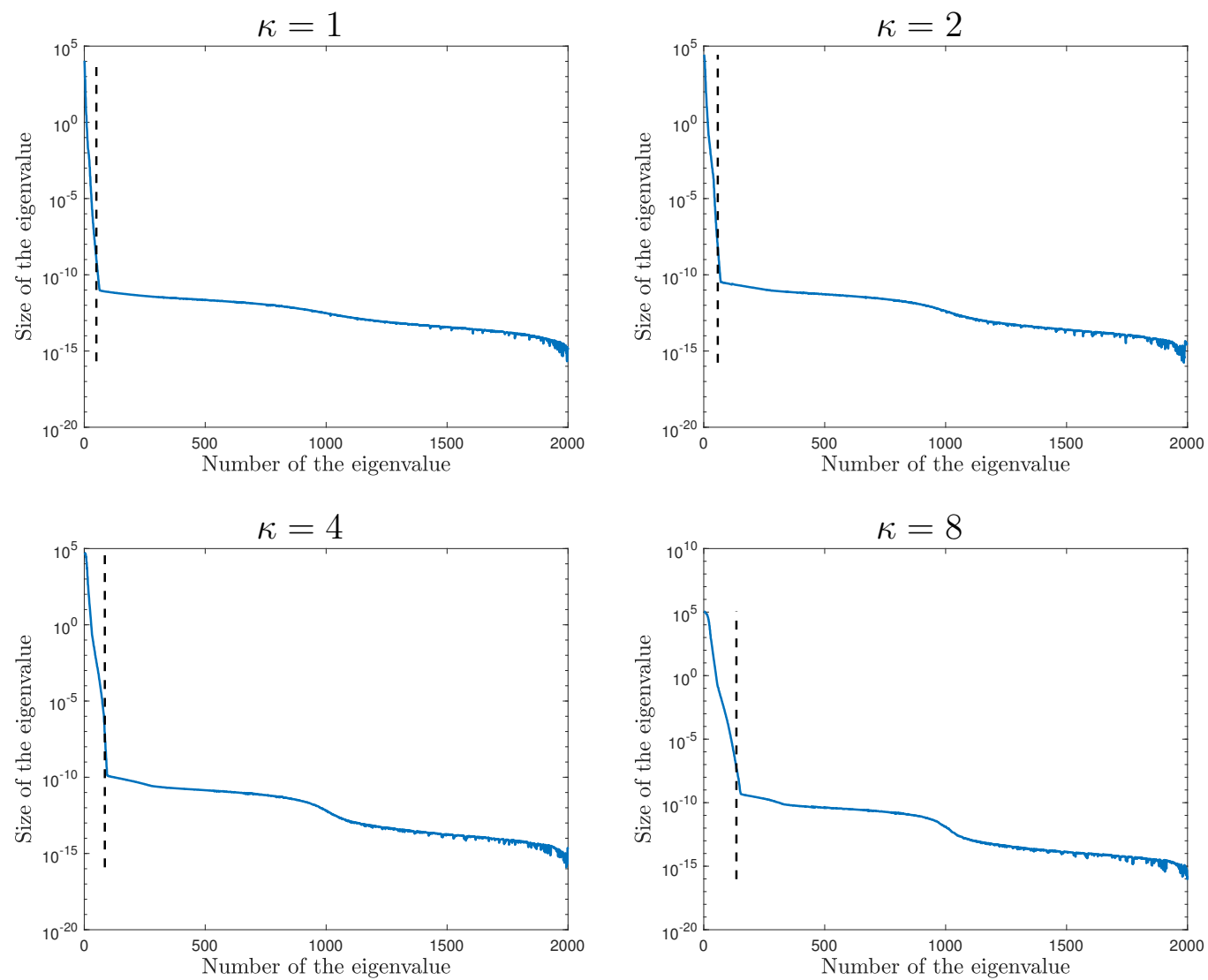

Figure 2: Decay of the eigenvalues of the covariance matrix $\mathbf{C}$ for the wavenumbers $\kappa=$ $1,2,4,8$ and $R=11$.

(4.3) and (4.6) using (5.7), where the incident wave comes again from the left, i.e., $\mathbf{d}=[1,0]^{\top}$. The results are found in Figure 3. For comparison, the scattered wave in case of the unperturbed kite-shaped scatterer is found in the first column. In the second column, the expected total wave is found. Finally, the variance of the total wave is found in the third column. The rows correspond to the wavenumber: the first row corresponds to $\kappa=1$, the second row corresponds to $\kappa=2$, the third row corresponds to $\kappa=4$, and the fourth row corresponds to $\kappa=8$.

One observes that, compared to the total wave of the unperturbed scatterer, the expected total wave is blurred towards the left, i.e., in directions opposite to the direction of the incoming wave. This is caused by the dif- 
ferent reflections at the perturbed scatterer which interfere. In the shadow region, i.e., towards the right, the expected total wave and the total wave of the unperturbed scatterer basically coincide. This observation is also underpinned by the variance of the total wave, which is maximal on the left of the scatterer and nearly 0 in the shadow region. Notice that the described smoothing effect becomes stronger as the wavenumber increases.

\subsection{Direction of the incident wave}

In the previous paragraph, we have computed the second order statistics of the scattered wave for varying wavenumber, while the incident wave was always fixed to $\mathbf{d}=[1,0]^{\top}$. Now, we shall fix the wavenumber to $\kappa=2$ and

consider different directions of the incident wave. The results are depicted in Figure 4. Here, the first row shows the total field for the nominal scatterer, the expected total wave for a random scatterer and its variance for an incident wave from the right in the first row, for an incident wave from the bottomright in the second row, for an incident wave from the bottem in the third row and finally for an incident wave from the bottom-left in the last row. It turns out that the total field is mostly affected by the perturbation of the scatterer in the direction of the incident wave. In particular, we observe a high variance, where the incoming wave hits the scatterer, while the variance is nearly zero in the shadow region.

\subsection{Far-field pattern}

We shall next consider the far-field pattern of the randomly perturbed kite-shape scatterer for the wavenumbers $\kappa=1,2,4,8$. The far-field has been evaluated in $n=1000$ equidistant points on $\mathbb{S}^{1}$. The results of the computations are found in Figure 5, where we have depicted the expected farfield (blue line) and the standard deviation of the far-field (dash-dotted line). We observe that the expected far-field pattern only oscillates in the shadow region. More precisely, on the left of the scatterer, i.e. $\pi / 2<\varphi<3 \pi / 2$, the average perturbed far-field does not follow the oscillations in the far-field of the nominal geometry (red line). This is in contrast to the deep shadow range, obtained for $\varphi \approx 0$, where the average far-field and nominal far-field are rather close to each other.

\section{Conclusion}

In the present article, we have proposed an efficient method for the computation of far-field statistics for acoustic scattering in the case of random 
obstacles. We have employed the Karhunen-Loève expansion to parametrize the random scattering problem with respect to the infinite dimensional hypercube. Then, the parametric scattering problem has been reformulated by means of boundary integral equations. This approach directly leads to a reduction of the spatial dimensionality from $d$ to $d-1$ and, consequently, to a reduction of the computational cost.

For the rapid computation of far-field statistics, like the mean and the variance of the far-field pattern, we have introduced an artificial interface, which almost surely contains all realizations of the random scatterer. Using this approach, all information of the random domain perturbation is assessable from the scattered wave's Cauchy data at this artificial interface. In particular, expressions for the far-field's or the scattered wave's expectation and variance can easily be derived. In order to speed up the computation of the variance, which is based on the evaluation of the covariance, we have suggested the application of a low-rank approximation method, namely the pivoted Cholesky decomposition.

The presented approach can also be formulated for the scattering at sound-hard obstacles. Moreover, it can be extended to other boundary value problems for which a Green's function is available. The observation that the solution's second order statistics is determined by the second order statistics of the Cauchy data on a deterministic interface holds even for arbitrary second order elliptic boundary value problems.

Numerical results have been provided for $d=2$. Here, the spatial discretization has been performed by the exponentially convergent Nyström method, while the quadrature in the random parameter is approximated by the Halton sequence. The numerical approximation of the Cauchy data for $d=3$ can be facilitated with linear cost in terms of degrees of freedom by a fast boundary element method, like e.g. fast multipole, see [8], or wavelets, see [13]. Since the evaluation of the correlation always involves the evaluation of the potential, a fast algorithm is required here as well. This can also be realized by the use of the fast multipole method, see [21].

\section{Bibliography}

[1] H. Brackhage and P. Werner. Über das Dirichletsche Außenraumproblem für die Helmholtzsche Schwingungsgleichung. Arch. Math., 16:325329, 1965. 
[2] R. Caflisch. Monte Carlo and quasi-Monte Carlo methods. Acta Numer., $7: 1-49,1998$.

[3] C. Canuto and T. Kozubek. A fictitious domain approach to the numerical solution of PDEs in stochastic domains. Numerische Mathematik, 107(2):257-293, 2007.

[4] J. E. Castrillon-Candas, F. Nobile, and R. F. Tempone. Analytic regularity and collocation approximation for elliptic $\{\mathrm{PDEs}\}$ with random domain deformations. Comput. Math. Appl., 71(6):1173-1197, 2016.

[5] D. Colton and R. Kress. Inverse Acoustic and Electromagnetic Scattering. Springer, Berlin-Heidelberg-New York, 2 edition, 1997.

[6] R. N. Gantner and M. D. Peters. Higher order quasi-monte carlo for baysian shape inversion. SIAM/ASA J. Uncertain. Quantif., 6(2):707736, 2018.

[7] N. Georg, D. Loukrezis, U. Römer, and S. Schöps. Uncertainty quantification for an optical grating coupler with an adjoint-based Leja adaptive collocation method. arXiv:1807.07485, 2018.

[8] L. Greengard and V. Rokhlin. A fast algorithm for particle simulations. J. Comput. Phys., 73(2):325-348, 1987.

[9] A.-L. Haji-Ali, H. Harbrecht, M. D. Peters, and M. Siebenmorgen. Novel results for the anisotropic sparse grid quadrature. J. Complexity, 2018.

[10] H. Harbrecht, M. Peters, and R. Schneider. On the low-rank approximation by the pivoted cholesky decomposition. Appl. Numer. Math., 62(4):428-440, 2012.

[11] H. Harbrecht, M. Peters, and M. Siebenmorgen. Efficient approximation of random fields for numerical applications. Numer. Lin. Algebra Appl., 22:596-617, 2015.

[12] H. Harbrecht, M. Peters, and M. Siebenmorgen. Analysis of the domain mapping method for elliptic diffusion problems on random domains. Numer. Math., 134(4):823-856, 2016. 
[13] H. Harbrecht and R. Schneider. Wavelet galerkin schemes for boundary integral equations -implementation and quadrature. SIAM J. Sci. Comput., 27(4):1347-1370, 2006.

[14] E. Hille and R. S. Phillips. Functional analysis and semi-groups. American Mathematical Society, Providence, 1957.

[15] R. Hiptmair, L. Scarabosio, C. Schillings, and Ch. Schwab. Large deformation shape uncertainty quantification in acoustic scattering. Adv. Comput. Math., 2018. to appear.

[16] C. Jerez-Hanckes, Ch. Schwab, and J. Zech. Electromagnetic wave scattering by random surfaces: Shape holomorphy. Math. Models Methods Appl. Sci., 27(12):2229-2259, 2017.

[17] R. Kress. Linear Integral Equations. Applied Mathematical Sciences 82. Springer, New York, 3 edition, 2014.

[18] M. Loève. Probability theory I+II. Number 45 in Graduate Texts in Mathematics. Springer, New York, 4 edition, 1977.

[19] M. D. Multerer. A note on the domain mapping method with rough diffusion coefficients. arXiv:1805.02889, 2018.

[20] H. Niederreiter. Random Number Generation and Quasi-Monte Carlo Methods. Society for Industrial and Applied Mathematics, Philadelphia, 1992.

[21] G. Of, O. Steinbach, and P. Urthaler. Fast evaluation of volume potentials in boundary element methods. SIAM J. Sci. Comput., 32(2):585602,2010 .

[22] X. Wang. A constructive approach to strong tractability using quasiMonte Carlo algorithms. J. Complexity, 18:683-701, 2002.

[23] D. Xiu and D. M. Tartakovsky. Numerical methods for differential equations in random domains. SIAM Journal on Scientific Computing, 28(3):1167-1185, 2006. 


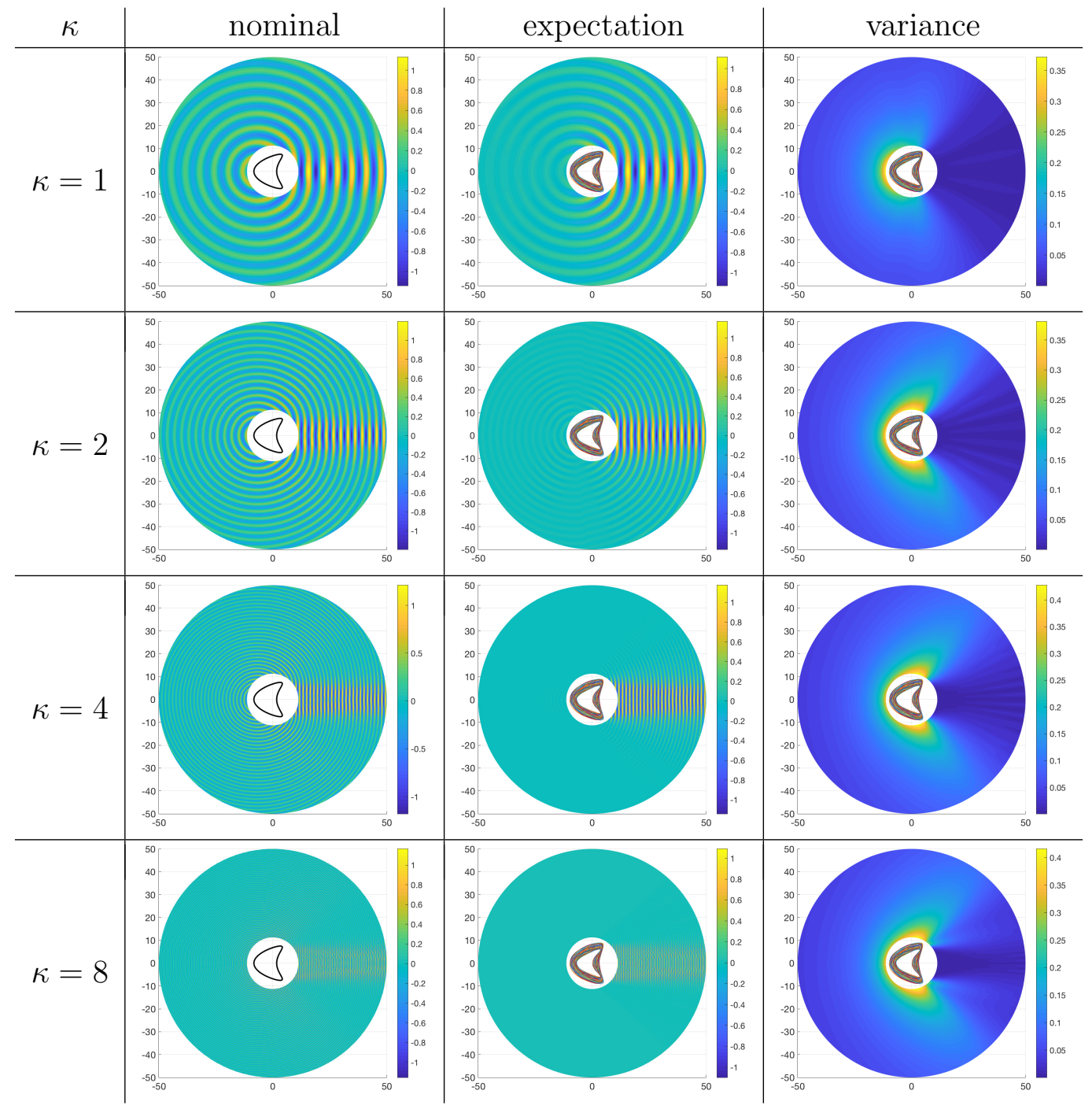

Figure 3: The total field of the nominal scatterer (left), the expected total field for the random scatterer (middle) and associated variance (right) for the wavenumbers $\kappa=1,2,4,8$. 


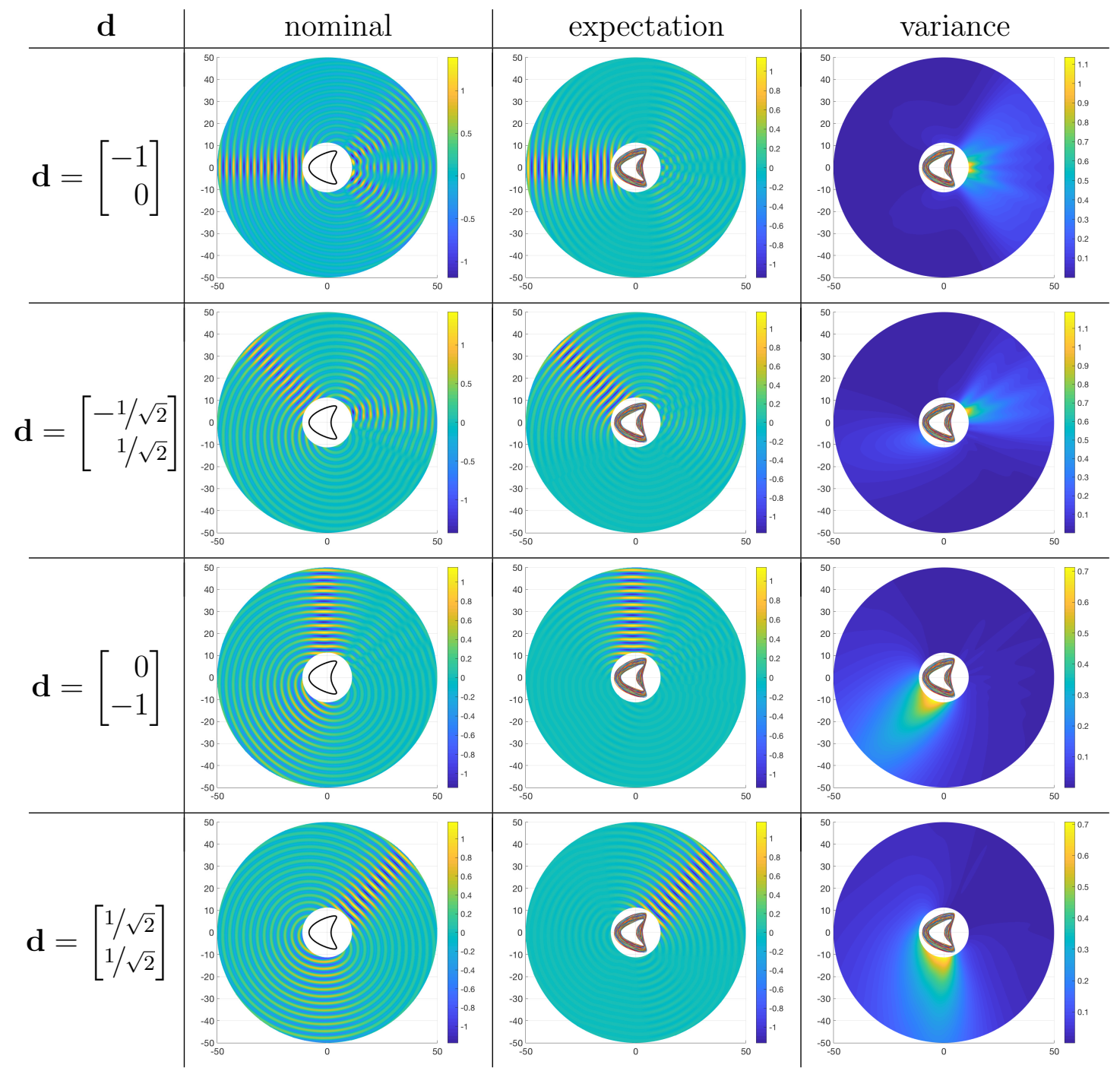

Figure 4: The total field of the nominal scatterer (left), the expected total field for the random scatterer (middle) and associated variance (right) for different directions of the incident wave. 

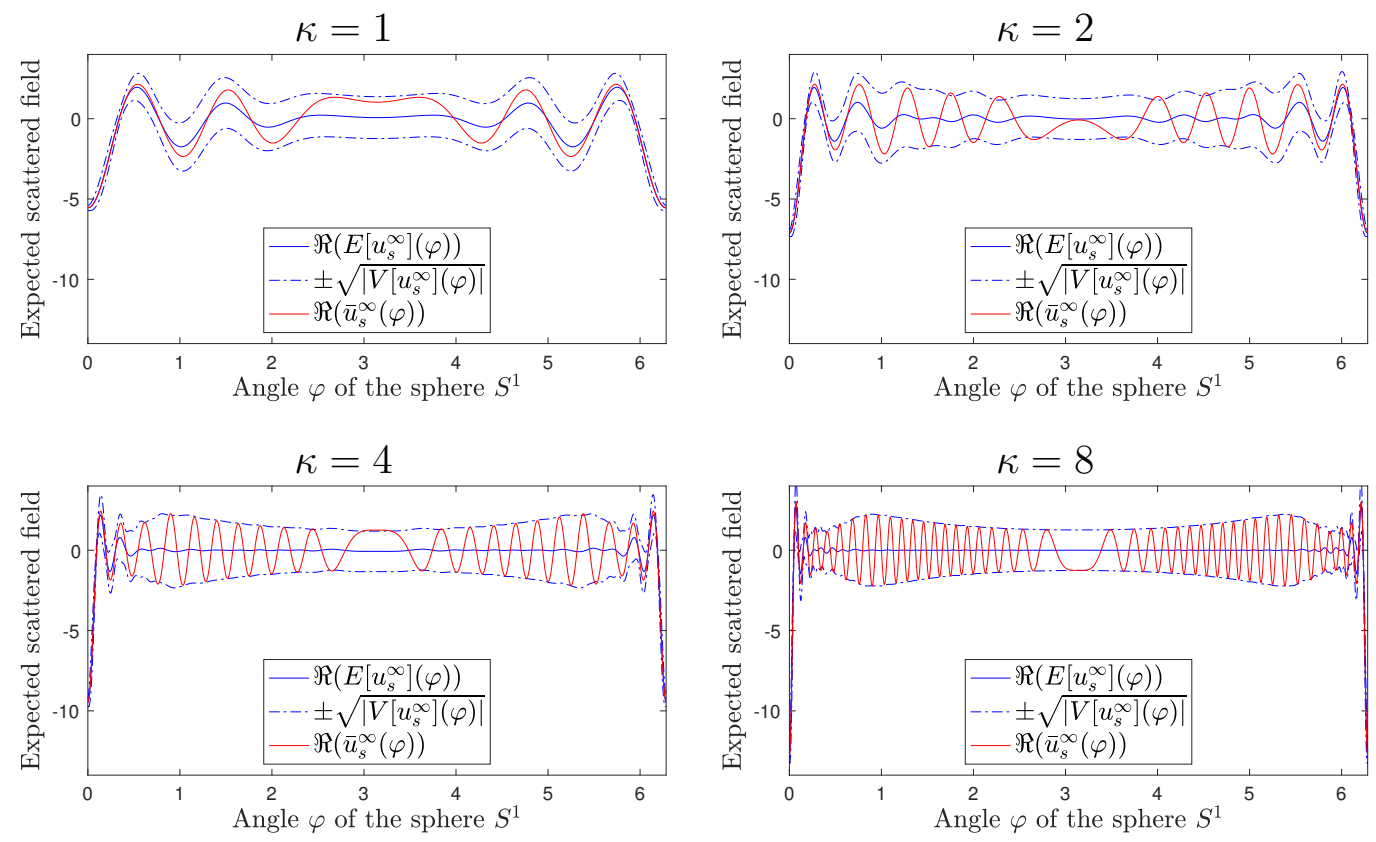

Figure 5: The real part of the expected far-field (blue line) with standard deviation (blue dash-dotted line) and the far-field of the unperturbed kite shape (red line) for $\kappa=1,2,4,8$. 